\title{
Contingent information processing: Contingent and precued classification
}

\author{
W. R. GARNER \\ Yale University, New Haven, Connecticut
}

\begin{abstract}
An experimental paradigm of contingent classification is introduced, in which stimuli in a classification task are cued by the addition of another dimension so that the classification is reduced to the discrimination of just two stimuli. The contingencies are arranged so that the cuing dimension provides no response information. Equivalent contingencies can be arranged by precuing in advance of the stimulus. Four experiments explored this paradigm with a discrete reaction task, using stimuli C, O, L, and T. In Experiment 1, with no additional cuing dimension, it was established that classification and discrimination based on the curved-straight distinction are easier than those not permitting its use. In Experiment 2, color was added as a cuing dimension, with both easy and hard classification tasks, in the latter case using contingencies that produced easy or hard discriminations. There was no improvement in performance with any of the contingent classifications. In Experiment 3, lateral location was used as a cuing dimension with a hard classification task, and in this case performance improved with a contingency that produced easy discriminations, but performance was not nearly as good as with the equivalent discrimination tasks alone. In Experiment 4, precuing of $500 \mathrm{msec}$ was used with a hard classification task, and performance improved almost to the level of the equivalent tasks, for both easy and hard discriminations. It is suggested that the use or nonuse of the contingent relations is best explained as due to the use of optional attentional allocation.
\end{abstract}

Most experiments concerned with the processing of relatively simple stimulus dimensions have involved either single dimensions or orthogonal combinations of dimensions, and in the latter case all dimensions may have equal status in defining the response assignments and thus the required nature of the information processing task. Yet, in a real world, even with relatively simple stimulus dimensions, most information processing is carried out in a contingent manner; that is, the attributes, features, or dimensions to be processed are determined by the level of some other stimulus property. Although, as Biederman (1972) pointed out, contingent processing is of central importance in most theories of complex information processing, very little investigation of contingent information processing has been done with relatively simple stimuli. That condition was true in 1972, and it has changed very little in the intervening years. The present paper first distinguishes several types of contingent information processing experiments and, second, presents some experiments exploring two types of contingent processing that have been used very little. The initial description of types of contingent processing follows Garner (1983).

\section{Contingent S-R Mapping}

One of the earliest experiments to study contingent relations (Shaffer, 1965) used one stimulus dimension whose

This research was supported by National Institute of Mental Health Grant MH 14229 to Yale University. Carolyn Paul carried out all of the experiments and did most of the data analysis. Her invaluable assistance is gratefully acknowledged. Requests for reprints should be sent to W. R. Garner, Department of Psychology, Box 11-A Yale Station, New Haven, CT 06520-7447. levels specified the stimulus-response mapping to be used with another dimension. Shaffer used a discrete reaction task to two lights placed to the left or right of center, with left and right keypresses as responses. Between the two lights there was another stimulus, a vertical or horizontal line, and the orientation of this stimulus specified which response was to be given to which of the two light stimuli, with either a homolateral or contralateral mapping being required. Biederman (1972) noted that such a task is really a biconditional task in which a single response is specified by the joint levels on the two dimensions. That this interpretation is correct is made clear by the fact that the line stimulus could be presented before or after the light stimulus, with essentially equivalent results. In other words, either stimulus dimension could be responded to contingent on the prior level of the other. Nevertheless, this experiment is truly one involving contingent information processing, with either dimension being able to have its $S-R$ mapping contingent on the level of the other.

Two other early experiments were not strictly concerned with S-R mapping, but were concerned with the role of response assignments in determining the ability of a stimulus dimension to interfere with the processing of other dimensions. Both Hodge (1959) and Montague (1965) showed that in selective attention tasks in which some dimensions are relevant and others irrelevant for responding, interference was greater if the irrelevant dimensions had been used as sometimes relevant. Hodge, in particular, also made some dimensions relevant on the basis of the levels of other dimensions, and such a procedure is one involving contingent relations. These three experiments, however, although involving contingent stimulus 
relations, were primarily concerned with the role of the response in the tasks rather than in the contingent stimulus relations themselves.

\section{Contingent Identification}

Biederman (1972) was far more concerned with the nature of the stimulus contingencies in his experiment. Although, strictly speaking, his experiment was a classification task, because more than a single stimulus was used for a particular response, I have called his contingent task one of identification because the task could have been carried out with one stimulus per response. Furthermore, the logical structure of his experiment illustrates one way in which a contingent task can be set up, and it need only require identification. He used three stimulus dimensions: color, size of a circle, and angle of a diameter within the circle, with each dimension having two levels. One dimension (which I shall call the cuing dimension) specified which of the other two dimensions (which I shall call contingent dimensions) needed to be processed. An actual set of four possible stimuli might be: red large, red small, green left tilt, green right tilt. Thus, color was the cuing dimension, and if red, the dimension of size was to be processed; if green, the dimension of tilt was to be processed. Thus, which contingent dimension was to be processed was specified by the cuing dimension. Four responses were required, one for each stimulus. Although Biederman actually allowed the third dimension to occur at each of its levels, that procedure is not necessary to produce a contingent identification task. Biederman's results showed that subjects could perform a task with such contingent dimensions almost as easily as a task in which just two dimensions orthogonally combined were always relevant, and much more easily than a task in which knowledge of all three dimensions was necessary for correct identification.

This task represents one of two major ways in which the contingency can be established. In the first case, as used by Biederman, the stimuli are formed from nested dimensions, in which each of two contingent dimensions is nested within one level of the cuing dimension. In such an arrangement, knowledge of both the cuing and contingent dimensions is necessary for correct identification. In the second case, the contingent task would have four levels (one for each response) of one contingent dimension, for example, circle, square, triangle, cross; when the cuing dimension is added, we might have red circle, red square, green triangle, green cross. In this arrangement, which involves nested levels, actual knowledge of the cuing dimension is not necessary, since there is sufficient information in the contingent dimension to make one of the four responses. That is not to say, however, that the addition of the cuing dimension would not improve performance. For any pair of cuing and contingent dimensions, that is an experimental question.

There is, for present purposes, one important aspect to either the nested dimensions or the nested levels form of contingency: In both cases, the cuing dimension gives direct response information. With nested dimensions, the cuing dimension reduces the number of response alternatives from four to two, and although the two nested dimensions together provide the rest of the needed information to specify the response, neither alone provides as much information as does the cuing dimension. In similar fashion, with nested levels, the cuing dimension reduces the number of response alternatives from four to two, and although the contingent dimension provides the rest of the needed information, it does so with two different subsets of levels. Thus, experiments of this type, concerned with the perception and use of contingent stimulus properties, are confounded by the direct response information provided by the cuing dimension.

\section{Contingent Classification}

A modification of the contingent identification task, however, can provide contingent stimulus relations in such a way that the cuing dimension gives no response information. The task is classification, in which more than one stimulus is assigned a single response, and the cuing dimension is used to provide a contingent relation so that each level of the cuing dimension leads to each of the possible responses, thus providing no direct response information.

An illustration of such a task, with some of the stimuli used in the present experiments, is given in Table 1. There are four levels of the contingent dimension (letter or form), and these are assigned to responses with a 4:2 mapping. This task is a straightforward classification task. However, color is added as a cuing dimension so that each level of color specifies (cues) two of the four stimuli. In the actual example, on trials when red occurs, only the letters $\mathrm{C}$ and $\mathrm{O}$ can occur. Conversely, on trials when green occurs, only the letters $\mathrm{L}$ and $\mathrm{T}$ can occur. With the proper response assignment, as shown, the cuing dimension gives no direct response information. There are, of course, just three ways in which four stimuli can be placed into two classes of two each. The addition of a dichotomous cuing dimension does not increase the number of classification tasks that are logically different because of the constraints of there being just two classes of stimuli and just two responses. However, with a judicious choice of stimuli and contingent assignments, various questions about the nature of contingent classification can be asked.

What is labeled in Table 1 as the contingent dimension need not be a single dimension, and, in fact, it is not clear

Table 1

A Contingent Classification Task in Which Four Levels of a Contingent Dimension Are Changed to Two Groups by a Cuing Dimension, but with the Cuing Dimension Providing No Response Information

\begin{tabular}{lcc}
\hline \multicolumn{2}{c}{ Dimension } & \\
\cline { 2 - 3 } Cuing & Contingent & Response \\
\hline Red & $\mathrm{C}$ & 1 \\
Red & $\mathrm{O}$ & 2 \\
Green & $\mathrm{L}$ & 1 \\
Green & $\mathrm{T}$ & 2 \\
\hline
\end{tabular}


whether the four letters, C, O, L, T are themselves a single dimension. The letters $\mathrm{C}$ and $\mathrm{O}$ share the property of curvature, but differ in whether the curved letter is open or closed; likewise, the letters $\mathrm{L}$ and $\mathrm{T}$ share the property of being straight lines, but differ in whether the horizontal line is at the top or bottom. Thus, these four letters can themselves be considered as consisting of two dimensions (open-closed, top-bottom) nested within the levels of the curved-straight dimension. That is to say, the set of letters considered as a single contingent dimension could actually be considered a contingent classification task in and of themselves. Even further, however, it is quite possible to have dimensions nested within the cuing dimension of color used in Table 1. Red could cue large or small circle and green could cue dark or light circle, and with both size and lightness, the value of the other dimension would be held constant. And, of course, the contingent dimension could be simply four levels on a single dimension, not easily redefined as two or more other dimensions. Shape is commonly used as such a dimension, for example, with circle, square, triangle, cross as four levels on a single dimension. Thus, a cuing dimension can be added to many different types of nominally contingent dimensions.

A last comment concerns what the addition of a cuing dimension logically does to the classification task, regardless of the nature of the contingent dimension itself: It changes a simple 4:2 (in this case) classification task into a randomly alternating pair of discrimination tasks. Thus, in Table 1 , if the cuing information provided by the color red is used by the subject, the task is a discrimination between $\mathrm{C}$ and $\mathrm{O}$; and if the color is green, the discrimination task is between $\mathrm{L}$ and $\mathrm{T}$. Since classification tasks are nearly always more difficult than two-choice discrimination tasks, the effective use of the cuing dimension should improve any measure of performance. The exception to this statement would be a set of contingent stimuli that can allow selective attention to one or more properties of the stimuli so that the classification task is not, in fact, more difficult than the equivalent discrimination tasks.

\section{Precued Classification}

If the information provided by the cuing dimension is presented in advance of the stimulus from the contingent dimension, all the logical properties of the contingent classification still hold, and all the ways in which the contingent dimension can vary also still hold. The only difference is in giving advance information of the sort that changes the classification into one discrimination task on each trial. The precuing technique has been widely used in recent years (LaBerge, Van Gelder, \& Yellott, 1970; but see Posner, 1978, for a variety of uses). Usually the technique is simply called cuing, but since I want to differentiate between cuing simultaneously with the stimulus presentation and cuing in advance, I shall distinguish between cuing and precuing.
The most common procedure is to cue a single stimulus from the set of possible stimuli, since such cuing is appropriate to the questions usually being addressed. Furthermore, the cuing is probabilistic, with the cue being valid on some trials and invalid on others. The probabilistic procedure is necessary with single stimulus precuing because otherwise the precue would give complete response information, and the actual stimulus presented later is not even necessary. Such precuing of single stimuli, however, gives many types of information: information is provided about the particular stimulus, its stimulus class, and the response. In fact, LaBerge et al. (1970) found that the single stimulus cue improved performance for both the correct modality and the correct response, even when the cue was invalid for the actual stimulus itself.

Ruth (1976; also described in Garner, 1980) used a technique of precuing which also involved probabilistic precuing but otherwise conformed to the requirements given here for contingent classification in some of her conditions. Her stimuli were dot patterns differing in configural goodness, and when she cued figural goodness, improvement in speed of performance was obtained even though the cue, when valid, did not provide any response information. More recently, Miller (1983) used precuing (in his Experiment 4) in which right and left responses were required to four letters, of which two appeared in an upper location and two in a lower location. He found a moderate improvement in classification when an arrow precued (by 1,500 $\mathrm{msec}$ ) whether the letter would be in the upper or lower position. This task, as used by Miller, is completely analogous to the contingent classification task with precuing, although for Miller it was simply a control condition, since his interest was primarily in response preparation.

When precued classification is used, the range of questions that can be investigated is expanded, since different properties of the set of stimuli can be precued, and some may be more effectively precued than others. As one example, in the Ruth (1976) experiment, the very fact that a stimulus property of configural goodness was capable of being precued effectively allows us to infer that the subject does, indeed, use that property of the stimulus in perceiving the entire set of stimuli. Furthermore, in Table 1 , it could be of interest whether it is better to precue pairs of letters that share curvature or straightness or to precue letters that differ in that respect. In the former case, the pair precued would be easy to remember but difficult to discriminate; in the latter case, the pair would be more difficult to remember because the stimuli do not share a property, but easy to discriminate for that very reason.

\section{Purpose of Experiments}

The four experiments reported explored the use of contingent and precued classification. The first experiment used the letters $\mathrm{C}, \mathrm{O}, \mathrm{L}, \mathrm{T}$ alone, since they can be considered to provide contingent classification by themselves, 
and to provide baseline data for the additional experiments. The second experiment used color cuing of the classification tasks, with all three of the possible ways of doing so. The third experiment used location cuing of a hard classification task so as to produce easy and hard discrimination tasks. The fourth experiment used simple precuing (actual specification of the letter pair in advance) to determine the limit to which cuing can be effective with these stimuli.

\section{General Methods}

\section{Stimuli}

The stimuli for all experiments were the letters $\mathrm{C}, \mathrm{O}, \mathrm{L}$, and $\mathrm{T}$, presented in a Scientific Prototype tachistoscope, for which $12.7 \times 17.8 \mathrm{~cm}$ cards were used to display the stimuli, one stimulus per trial. The stimuli were viewed binocularly at $127 \mathrm{~cm}$. The stimuli were black letters on a white background (with one minor exception). The background luminance was $17.1 \mathrm{~cd} / \mathrm{m}^{2}$.

The actual stimuli used are displayed in Figure 1. The letters were Letraset 725, 30-pt Helvetica Medium. These particular letters were used because two of them are composed only of curved lines and the other two are composed only of straight lines, with no serifs or other flourishes that would prevent a clear distinction between curved and straight letters. The actual physical measurements of the $O$ (the widest letter) were $8.0 \mathrm{~mm}$ high and $7.5 \mathrm{~mm}$ wide, with a line thickness of $1.6 \mathrm{~mm}$. At the viewing distance of $127 \mathrm{~cm}$, the visual angle of the height was $.36^{\circ}$.

\section{Procedure}

A single trial commenced when the subject pushed a button switch held in the left hand, at which time a 500-msec interval occurred before the stimulus presentation. This $500-\mathrm{msec}$ interval contained different things in the four experiments. The stimulus remained on for $1,000 \mathrm{msec}$, and the subject responded by moving a small switch lever mounted under the tachistoscope, up or down according to the specific task and instructions. Whatever the task, the actual
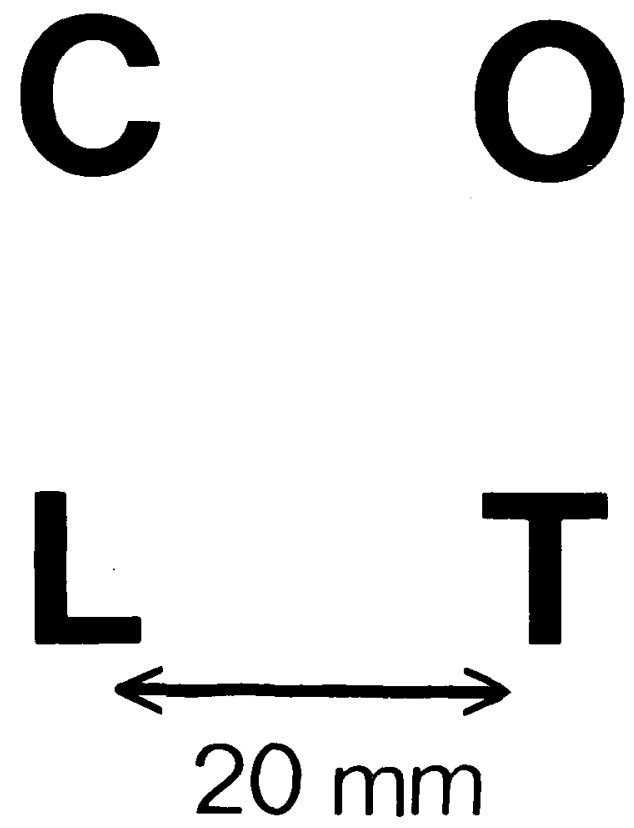

Figure 1. The four stimuli used in all of the experiments. stimulus-response assignments were reversed for half the subjects. A computer recorded the response and the reaction time (RT), and also randomized the stimulus presentation orders. The stimulus cards were changed after the response had been made, and the sound of the card insertions into the tachistoscope was the signal to the subject that a new trial could be started. The total time for a single trial (including the intertrial interval) was about $6 \mathrm{sec}$.

Reminder cards. In all experiments except precuing, at all times between the starting of a trial and the ending of the stimulus presentation, a card was visible with a fixation dot in its center and with smaller letters $(5 \mathrm{~mm}$ high) located $20 \mathrm{~mm}$ above and below the level of the fixation dot (measured to the center of the letter). These letters indicated the response assignments for the particular task, and thus served as reminders of the assignments at any time during an experimental run. All letters appropriate to a particular task were on the reminder card.

Subjects were run in at least two sessions of approximately 50$60 \mathrm{~min}$. Sessions were run on different days except on rare occasions, and if two sessions were run on a single day, the sessions were separated by at least $1 \mathrm{~h}$.

\section{Data Analysis}

Errors. Error rates were small for all four experiments, ranging overall from $2.5 \%$ to $3.6 \%$ for the four experiments. Furthermore, within each experiment, the product moment correlation between error rates and RTs was positive, ranging from .27 for the colorcuing experiment to $.66, .73$, and .73 for the other experiments. The color-cuing experiment had the smallest range of RTs and errors because only classification tasks (no discrimination tasks) were used in that experiment. Thus, the one moderately small correlation was due to a restricted range of scores. In none of the experiments, however, is there any evidence of a speed-accuracy tradeoff. That fact, plus the small number of errors, means that little can be gained by further analysis of errors, and they will not be discussed in each experiment.

Reaction times. The primary dependent value is therefore RT. For each block of trials for each subject, a median RT was computer calculated for each stimulus (correct responses only), the median being used to minimize the effects of outliers. All further analyses were done with means of these original median scores. Analyses of variance (ANOVA) were then used for further analysis, with various contrast tests to address specific questions.

\section{EXPERIMENT 1: C, O, L, T}

\section{Subjects}

\section{Method}

Nine subjects, recruited from Yale students and staff, were paid for participation. All had normal or corrected vision, and none had been used in this type of research before.

\section{Tasks}

Classification. There are three possible classification tasks that can be used with four stimuli, and all three were used: CO/LT, $\mathrm{CL} / \mathrm{OT}$, and CT/OL. The slash indicates the appropriate stimulus groupings for each of the two responses. Note that only the CO/LT task allows use of the curved-straight distinction as a basis of classification.

Discrimination. Only two stimuli are used in the discrimination tasks, with a different response for each stimulus. There are six different pairings of the six stimuli possible, and all were used: $\mathrm{C} / \mathrm{O}$, $\mathrm{C} / \mathrm{L}, \mathrm{C} / \mathrm{T}, \mathrm{O} / \mathrm{L}, \mathrm{O} / \mathrm{T}$, and $\mathrm{L} / \mathrm{T}$. Note that four of the discrimination pairs allow use of the curved-straight distinction, while two pairs, $\mathrm{C} / \mathrm{O}$ and $\mathrm{L} / \mathrm{T}$, require discrimination within the curved-straight distinction, that is, require that one of the nested dimensions be the basis of discrimination. 


\section{Stimulus Presentation}

When the subject initiated a trial, a gray field (Pantone 431-A, Munsell equivalent 5B 5.5/1) appeared immediately. In the center of the gray field there was a $17-\mathrm{mm}$ square opening exposing the white card underneath. At the end of the 500 -msec interval, the stimulus letter appeared, centered in the square opening of the gray field, black on white, for the duration of the stimulus exposure, at which time the stimulus field reverted to the card with the fixation dot and response assignment. The reminder letters were centered for discrimination tasks and were separated horizontally by $25 \mathrm{~mm}$ (center to center) for the classification tasks.

\section{Experimental Design}

The nine subjects and tasks were arranged in a Latin square so that each classification task was used in each third of the order of the nine tasks, but never was followed or preceded by another classification task. Furthermore, across subjects, no pair sequence of tasks occurred more than twice. All nine tasks were used in a single session, and each task had a total of 60 trials, half for each response. Thus, for each stimulus letter, there were 15 trials per stimulus in the classification tasks and 30 trials per stimulus in the discrimination tasks. Before each task was started, 4 to 8 practice trials were given, at least 2 for each stimulus.

Each subject was run for two sessions, with the same Latin square. Data from the first session were considered practice, and data from the second session only were used.

\section{Results}

The tasks and mean RTs are shown in Table 2. An overall one-factor ANOVA showed the differences between conditions to be significant $[F(8,64)=31.79, p<.001]$. A specific contrast comparing the mean of the classification tasks ( $422 \mathrm{msec})$ with that of the discrimination tasks $(347 \mathrm{msec})$ was also significant $[F(1,64)=179.80$, $\mathrm{p}<.001]$. Since classification tasks are in general more difficult than discrimination tasks, this result was to be expected. Further analyses are concerned with specific aspects of these two types of tasks.

\section{Classification Tasks}

The CO/LT task allowed use of the curved-straight distinction, and its mean RT (373 msec) was $73 \mathrm{msec}$ faster than the mean RT $(446 \mathrm{msec})$ of the two classification tasks that had both a curved and a straight stimulus in each class $[F(1,64)=55.96, p<.001]$. These two mixed classification tasks did not differ from each other, a difference of $22 \mathrm{msec}$ being required between any pair of sin-

Table 2

Experiment 1: Reaction Time for All Possible Classification and Discrimination Tasks with the C, O, L, T Stimuli

\begin{tabular}{ccc}
\hline Task Type & $\begin{array}{c}\text { Specific } \\
\text { Task }\end{array}$ & $\begin{array}{c}\text { Mean RT } \\
\text { (msec) }\end{array}$ \\
\hline Classification & CO/LT & 373 \\
& CL/OT & 454 \\
& CT/OL & 438 \\
Discrimination & C/O & 361 \\
& C/L & 346 \\
& C/T & 339 \\
& O/L & 332 \\
& $\mathrm{O} / \mathrm{T}$ & 336 \\
& $\mathrm{~L} / \mathrm{T}$ & 368 \\
\hline
\end{tabular}

Note - The slash separates the stimuli to be given different responses. gle tasks for significance at the .05 level. Thus, the curved-straight distinction dominated the results of the classification tasks.

\section{Discrimination Tasks}

This same curved-straight distinction dominated the results of the discrimination tasks as well. The mean RT for the four tasks that allowed use of the curved-straight property was $338 \mathrm{msec}$, whereas that for the two tasks that required discrimination either between the curved letters or between the straight letters was $364 \mathrm{msec}$, a significant difference of $26 \mathrm{msec}[\mathrm{F}(1,64)=14.45$, $\mathrm{p}<.001]$. Neither the $\mathrm{C} / \mathrm{O}$ nor the $\mathrm{L} / \mathrm{T}$ tasks differed from each other, and none of the four tasks allowing use of the curved-straight distinction differed significantly either.

\section{Task Type Comparisons}

Even though the curved-straight distinction dominated results for both the classification and the discrimination tasks, there still are meaningful differences between the two types of task. The easy classification task and the easy discrimination tasks (that is, those allowing use of the curved-straight distinction) still differed from each other, the mean RT of $373 \mathrm{msec}$ for the classification task being significantly greater than the mean RT of $338 \mathrm{msec}$ for the four equivalent discrimination tasks $[F(1,64)=15.99, p<.001]$. This 35 -msec difference is approximately half that between the easy and hard classification tasks, and shows that even when a dominant property such as the curved-straight distinction is available, classification is still more difficult than discrimination.

This point is made again when the mean RT (446 msec) for the two hard classification tasks is contrasted with the mean RT (364 msec) of the two hard discrimination tasks (i.e., those not allowing use of the curved-straight distinction) $[F(1,64)=106.95, p<.001]$. This difference of $82 \mathrm{msec}$ is more difficult to interpret because the classification tasks involve discriminations both between and within the curved-straight distinction.

One last contrast does suggest, however, that the discrimination tasks and the classification tasks can be made equally easy or hard: The easy classification task (mean $\mathrm{RT}=373 \mathrm{msec}$ ) was slightly slower than the hard discrimination tasks (mean RT $=364 \mathrm{msec}$ ). This difference is not significant, and simply makes the point that discrimination may be as difficult as classification if an easy classification task is compared with a difficult discrimination task, even by selecting specific tasks from a small set of stimuli.

\section{Discussion}

As noted in the introduction, this set of stimulus letters can themselves be considered as providing contingent classification, since there are two dimensions nested within levels of the curved-straight dimension. If very rapid perception of whether a letter was curved or straight had occurred, then the subject had information about which two 
stimuli were the possible choices on each particular trial. That the stimuli were not processed in this manner is evident from the data on the classification tasks, in which the easy classification task that could use the curvedstraight distinction was $73 \mathrm{msec}$ faster than the two tasks in which use of the nested dimensions was required. This result seems reasonable, however, when the data from the discrimination tasks are considered, because these data make clear that discriminations between stimuli that differed only on the nested variable $(\mathrm{C} / \mathrm{O}$ and $\mathrm{L} / \mathrm{T})$ were considerably more difficult than those that allowed use of the curved-straight dimension. Thus, although subjects might have tried to use these stimuli as a contingent classification task, the two stimuli specified by the curvedstraight distinction were themselves difficult to discriminate.

The question of proper controls to determine whether contingent processing has been used at all is relatively simple. If contingent classification had been effectively used, then the classification tasks allowing its use (CL/OT and CT/OL) should have been as fast as the two discrimination tasks involving the nested dimensions that resulted with the use of the contingency. The fact that the results were not close to this expectation suggests that no use of the contingency was made.

The effectiveness of the curved-straight distinction is so overpowering in these results that they indicate almost complete selective attention to that dimension. From the classification data alone, it is tempting to suggest that selective attention was being used for that dimension. However, the equivalent discrimination data are still significantly faster than the classification task. So irrelevant variation in the nested dimensions was having some effect in the classification tasks.

\section{Color Cuing}

These results in and of themselves are consistent and reasonable, given the properties of the four letters used as stimuli, and these stimuli had been selected to provide an unequivocal curved-straight distinction. Thus, in effect the choice of these stimuli letters made the difference found in both types of task essentially inevitable. However, these differences make possible some particularly interesting combinations when a cuing dimension is

Table 3

Experiment 2: Reaction Times for Easy and Hard Classification Tasks, With and Without Contingent Cuing with Color

\begin{tabular}{lcc}
\hline Classification Type & $\begin{array}{c}\text { Specific } \\
\text { Task }\end{array}$ & $\begin{array}{c}\text { Mean RT } \\
\text { (msec) }\end{array}$ \\
\hline Easy & & \\
Control & CO/LT & 378 \\
Contingent & C/T,O/L & 395 \\
Hard & & \\
Control & CL/OT & 431 \\
Contingent & C/T,L/O & 424 \\
Contingent & C/O,L/T & 423 \\
\hline
\end{tabular}

Note - With the contingent classification tasks, the comma separates the two components of the classification that are cued by two different colors. added to the stimulus set. For example, with either of the hard classification tasks, cuing can be done so as to produce either hard or easy discrimination subsets of the stimuli, and if differences occur between these forms of cuing, then it will be clearer how contingent processing operates.

In Experiment 2, color will be used as a cuing dimension, with all of the three possible ways of arranging the levels of the cuing dimension with the classification tasks to produce contingent classifications.

\section{EXPERIMENT 2: COLOR CUING}

\section{Subjects}

\section{Method}

Ten subjects, recruited from Yale students and staff, were paid for participation; none had been used in this type of research before. All had normal or corrected vision and, before being used as subjects, all were given a simple color test in which they were required to select a good green and a good red from a random array of colors. All subjects were able to pass this color test.

\section{Tasks}

All tasks used were classification, two as controls and three as contingent classification. These are shown in Table 3.

Easy classification. Two of the tasks involved the easy classification from Experiment 1, CO/LT. The control task used no color cuing. The contingent task stimuli were presented with either a red or a green color that had the effect, if used, of reducing the number of alternatives on a given trial to two. Thus, if the letters $\mathrm{C}$ and $T$ were presented with red and the letters $O$ and $L$ were presented with green, in effect there were two randomly intermixed discrimination tasks whose nature was cued by color. The notation used for this contingent classification task is, as shown in Table 3 , $\mathrm{C} / \mathrm{T}, \mathrm{O} / \mathrm{L}$,

With the easy classification task, there are two different ways of assigning the contingent relations, but each of them leads to a pair of easy discrimination tasks, as indicated by the data of Experiment 1 . Thus, only one contingent assignment of color was used, and this task involves easy classification plus easy discrimination.

Hard classification. There were two hard classification tasks as indicated by the data of Experiment 1, but each of them allowed equivalent assignment of contingent relations. Therefore, only the CL/OT classification task was used. However, the two different ways of assigning contingent relations now lead to either easy or hard discriminations. The task $\mathrm{C} / \mathrm{T}, \mathrm{L} / \mathrm{O}$ has two easy discriminations with different colors assigned to each pair of stimuli involving the discriminations. On the other hand, the task $\mathrm{C} / \mathrm{O}, \mathrm{L} / \mathrm{T}$ has two difficult discriminations by the use of color cuing. Therefore, three tasks were used with the hard classification. One of them was the control, in which no color was used. One contingent task involved hard classification plus easy discrimination; the other contingent task then involved hard classification plus hard discrimination.

\section{Stimulus Presentation}

Color surround. Two different methods of presenting the color cuing were used. One of them was analogous to the stimulus presentation method of Experiment 1, in which the letter was surrounded by a gray field. With the color-surround method, the control classification was exactly as in Experiment 1, with a gray field containing a 17-mm square opening occurring for the $500 \mathrm{msec}$ at the initiation of the trial and with the stimulus appearing centered in the opening after the $500-\mathrm{msec}$ period. When color cuing was used, the gray field appeared during the 500 -msec period, but then changed to the appropriate color when the stimulus appeared in the open- 
ing. The two colors used were a highly saturated red (Pantone 032A, Munsell equivalent 5R 5.5/14) and green (Pantone 347-A, Munsell equivalent $2.5 \mathrm{G} \mathrm{5.5/12}$ ). With these values, the brightness did not change when the stimulus appeared, but the color of the surround did.

Color overlay. Instead of a surround, the second method used an overlay of color across the entire field, including the stimulus. In order not to mask the black stimulus, a much lighter color was necessarily used. With this method, the 500 -msec period at the beginning of a trial exposed a blank field, after which the stimulus plus color overlay appeared. For control classification, the overlay was gray (Pantone 429-A, Munsell equivalent Neutral 8/0). For red cuing, the overlay was Pantone 189-A (Munsell equivalent 7.5RP 8/8), and for green, it was Pantone 358-A (Munsell equivalent 7.5GY 8/6).

The actual color assignments to the particular pairs of letters were reversed for the two methods of color cuing. In addition, the reminder cards that were always available between trials contained small squares of the appropriate colors above or below the letters, indicating response assignments, above for the upper letters and below for the lower letters. These squares were $8.0 \mathrm{~mm}$ on a side and were located $7.0 \mathrm{~mm}$ from the nearest edge of the letter.

\section{Experimental Design}

Each subject was run for five sessions, and in each session a single task was used to allow for as much opportunity for learning to use the contingencies as possible. After a few practice trials to instruct the subject about the task for that session, five blocks of 120 trials were run, and since each task used all four stimuli, there were 30 trials per stimulus. The subjects were given 1- to 2-min breaks between blocks of trials. Data were used from the last two blocks only

Five subjects were assigned to each of the two methods of stimulus presentation. For each method of presentation, a Latin square was used to determine the order of tasks. Two different Latin squares were used and were arranged so that over the two squares each task was followed or preceded by each other task two times.

\section{Results}

The results are displayed in Table 3. A preliminary ANOVA was carried out to determine whether the two methods of stimulus presentation had any effect. The mean RT for the color surround method was $406 \mathrm{msec}$; that for color overlay was 414 . This difference is not significant, nor is the interaction between method of presentation and task. Therefore, Table 3 shows data pooled over the two methods of presentation and all further analyses were done with the pooled data.

\section{Classification Type}

An overall ANOVA showed that task was a significant factor $[\mathrm{F}(4,36)=12.25, \mathrm{p}<.001]$. However, a series of contrasts indicated that this significance was due entirely to the difference of $40 \mathrm{msec}$ between the means of the easy and hard classification types $[F(1,36)=44.91$, $p<.001]$. This factor alone accounted for $92 \%$ of the variance due to tasks. No contrasts between control and contingent classifications were significant, a fact that the data in Table 3 make fairly self-evident.

\section{Discussion}

This experiment is easily summarized: Color as a cuing dimension to provide contingent classification was completely ineffective. This experiment merely replicated the result of Experiment 1, which showed the difference between the easy and the hard classification tasks with the $\mathrm{C}, \mathrm{O}, \mathrm{L}, \mathrm{T}$ letters as stimuli. Furthermore, the full hour used for each task should have been sufficient practice to allow any effects to occur.

Color had been chosen as the cuing dimension because there is ample evidence in the literature that it may have some special status compared with such stimulus properties as size, brightness, form, etc. (see Garner, 1974, 1983, for a summary of this evidence). Of special interest for this type of task, however, is evidence from Von Wright (1968) that color can be used to designate the subset of letters to be reported in a partial-report procedure of the kind used to establish the existence of an iconic store, and that it is as effective as designating the subset by location, such as rows or columns. Thus, there was good reason to expect that color would be effective, but it was not effective in this experimental paradigm with these particular stimuli.

\section{Location Cuing}

As just noted, the other stimulus dimension likely to be most effective in the contingent task is location. It is the dimension most frequently used in any task in which dimensional attention is required, and that is because it seems most natural to use it, since stimuli must necessarily be distributed across visual space or retinal location. For example, Duncan (1981), in writing on selective attention, notes that selection is especially effective when based on advance knowledge of position. Tsal (1983) goes even further in noting that location information, unlike color or brightness, is always present and is thus a special dimension not like the others. One can attend to location without there being a stimulus present, and thus giving information about location can direct attention in a way that color cannot.

For these various reasons, the next experiment used lateral location as the cuing dimension in contingent classification.

\section{EXPERIMENT 3: LOCATION CUING}

\section{Method}

\section{Subjects}

Fourteen subjects were recruited from Yale students and staff and were paid for participation. All had normal or corrected vision. Seven of the subjects had had previous experience in this type of experiment, and seven had had none. These inexperienced subjects were given $1 \mathrm{~h}$ of practice on the tasks before being used in the experiment proper.

\section{Tasks}

Classification. The hard classification task used in Experiment 2, CL/OT, was used again. It was used in three forms. As a control, all four stimuli appeared randomly in the right and left locations. In two contingent tasks, two letters always appeared on the left and the other two always on the right, so that location was serving as a cuing dimension. Two assignments were used. In one, the two letters on each side provided an easy discrimination, $C / T, L / O$. In 
the other contingent task, the two letters on each side provided a hard discrimination task, $\mathrm{C} / \mathrm{O}, \mathrm{L} / \mathrm{T}$. Thus, all three classification tasks were hard classifications, but one contingent task had easy discriminations and the other had hard discriminations.

Discrimination. Because of the varying locations used in this experiment, the discrimination data obtained in Experiment 1 could no longer be considered adequate as control data. Therefore, four discrimination tasks were used, those corresponding to the equivalent cued discriminations in contingent classification. The easy discrimination tasks were $\mathrm{C} / \mathrm{T}$ and $\mathrm{O} / \mathrm{L}$; the hard discrimination tasks were $\mathrm{C} / \mathrm{O}$ and $\mathrm{L} / \mathrm{T}$.

\section{Stimulus Presentation}

Location. The centers of the letter stimuli appeared $11.25 \mathrm{~mm}$ to the right or left of fixation. At the viewing distance of $127 \mathrm{~cm}$, this distance corresponds to approximately $.5^{\circ}$ of visual angle. The difference between these right and left locations is easily perceived, yet foveal vision is maintained.

Reminder cards. With discrimination tasks, the letters indicating response assignments on the reminder card were centered horizontally, as in Experiment 1. Thus, no cue to actual location of the stimulus presented was available. With the control classification task, the two letters requiring an up or down response were centered on the reminder card, with one letter above the other. The letter closer to the fixation was in the same location as the single letter for discrimination tasks, and the additional letter was $11 \mathrm{~mm}$ higher or lower, center to center. This arrangement was used to keep the right and left locations from being misleading, since all four letters occurred randomly in either position. With contingent classification, the letters indicating response assignment were located above and below vertical center, as with the discrimination tasks, but were located horizontally to correspond exactly to the possible locations of the letters. In contingent classification, of course, only two letters appeared in each of the two possible locations, so the response assignment card also gave exact location information for the appropriate letters.

Timing. To minimize memory demands, when a subject initiated a trial, the reminder card remained in view for $400 \mathrm{msec}$, followed by a blank period of $100 \mathrm{msec}$, at which time the stimulus appeared for $1,000 \mathrm{msec}$.

\section{Experimental Design}

All seven tasks were used in each single session. For each of the three classification tasks, a total of 96 trials were used. With the control classification, with each stimulus appearing equally often at each location, these trials divided into 12 trials for each stimulus at each location. For the two contingent classification tasks, each stimulus appeared at only a single location, so that there were 24 trials per letter, with a fixed location for each letter. For each of the discrimination tasks, a total of 48 trials were used, and since these letters appeared randomly at both locations, there were 12 trials for each stimulus at each location. A few practice trials were given before each task to be sure the subject understood the task.

The seven tasks were run with a Latin square counterbalancing. The same Latin square was used for the seven experienced and the seven inexperienced subjects, but no further distinction was made between the two types of subject. Each subject was run for two sessions, but data were used from the last session only.

\section{Results}

The results are displayed in Table 4. For presentation and analysis of data, the seven tasks were reduced to five by averaging the results for the two easy and two hard discrimination tasks. These tasks do not differ within themselves, and by using average data for the tasks, they become directly comparable to their equivalent contingent tasks. Still further, because of the use of half as many
Table 4

Experiment 3: Reaction Times for Control Classification, Contingent Classifications Cued by Location, and the Equivalent Discrimination Tasks

\begin{tabular}{ccc}
\hline Task Type & $\begin{array}{c}\text { Specific } \\
\text { Task }\end{array}$ & $\begin{array}{c}\text { Mean RT } \\
(\mathrm{msec})\end{array}$ \\
\hline Classification & & \\
Control & CL/OT & 411 \\
Contingent & C/T,L/O & 400 \\
Contingent & C/O,L/T & 414 \\
Discrimination & C/T and O/L & 339 \\
& C/O and L/T & 364 \\
\hline
\end{tabular}

Note - The comma separates the two components of the classification tasks that are cued by different locations. RTs are averaged for the pairs of discrimination tasks that correspond to the two equivalent cued contingent tasks.

trials for the discrimination tasks as for the classification tasks, by combining the discrimination tasks they become comparable to their equivalent contingent tasks in total number of trials.

\section{Location Effects}

A preliminary analysis was done to determine whether the left or right position of the stimuli had any effect on RT. The overall mean for all stimuli occurring on the left was $386 \mathrm{msec}$, and that for those occurring on the right was $385 \mathrm{msec}$. Of the five tasks shown in Table 4, two showed a slight advantage for the left and three did so for the right. It thus is clear that actual right-left position was not a factor that needed further consideration in the analyses.

\section{Task Type Comparisons}

An overall ANOVA was carried out first, and it showed significant effects of tasks $[F(4,52)=58.14, p<.001]$. A contrast between the classification tasks (control and contingent) and the discrimination tasks was also significant $[\mathrm{F}(1,52)=208.84, \mathrm{p}<.001]$. Although this factor alone accounted for $90 \%$ of the variance due to tasks, there were significant effects within each type of task, and further analyses are concerned with these.

\section{Classification Tasks}

The RT of $411 \mathrm{msec}$ for the control classification task was not significantly different from the $414 \mathrm{msec}$ of the contingent task with hard discriminations $(\mathrm{C} / \mathrm{O}, \mathrm{L} / \mathrm{T})$. But the control classification task, contrasted to the easy contingent task $(\mathrm{C} / \mathrm{T}, \mathrm{L} / \mathrm{O})$ (11-msec difference), barely missed significance at the .05 level $[\mathrm{F}(1,52)=3.67]$. A contrast between the hard contingent task and the easy contingent task (14-msec difference) was significant $[F(1,52)=5.77, p<.025]$. Since this result is directionally predicted, this significant contrast between contingent tasks providing easy discriminations and those providing hard discriminations can be considered valid. Further evidence of its validity is that 12 of the 14 subjects showed the effect [ $p$ (binomial) $=.006$, one-tailed].

Thus, there is evidence that some contingent processing was occurring, but that it improved performance only 
when the resultant discriminations were easy and had no effect when the resultant discriminations were hard.

\section{Discrimination Tasks}

The results from the discrimination tasks completely confirm those found in Experiment 1. The discriminations based on the curved-straight distinction were easier than those requiring discrimination within the curved or straight distinction: the difference of $25 \mathrm{msec}$ provides a contrast of $F(1,52)=17.27, p<.001$.

Of interest is whether the 25 -msec difference between easy and hard discrimination tasks is significantly greater than the 14-msec difference between the same discriminations when they are imbedded in a contingent classification task. A direct contrast of the interaction between the hard-easy discrimination tasks and their equivalents in contingent classification failed to show significance $[\mathrm{F}(1,52)=1.53, \mathrm{p}>.10]$.

Thus, these results show that location contingency was operating sufficiently well to produce a difference between contingent tasks providing hard and easy discriminations equivalent to the difference obtained with the equivalent discrimination tasks themselves. Despite this evidence of the use of the contingent relations, the discrimination tasks were still much easier, with an average of $56-\mathrm{msec}$-faster RT than that obtained with the contingent tasks $[F(1,52)$ $=165.23, \mathrm{p}<.001]$.

\section{Discussion}

This experiment with location as the cuing dimension did give clear evidence that the contingent relations were influencing performance. However, the influence was small, having little effect in improving overall performance, but having an effect in allowing the difference between easy and hard discrimination tasks to show up when the tasks were cued by location.

The more important question is why there was so little overall improvement, despite the clear evidence that the contingency relation was operating. That there is ample room for improvement is indicated by the fact that the discrimination tasks, both in this experiment and in Experiment 1 , provide considerably faster performance than do the classification tasks. There are several possible factors that might be operating. One is that the subjects do process the cuing dimension first, but that this processing adds enough time to disallow any overall gain. Such an explanation would fit quite easily with the results of this experiment, since the difference between easy and hard discriminations showed up but with very little overall improvement in performance. It is as though the added processing time for the cuing dimension cancels out the savings in time for the contingent stimuli themselves. Such a notion implies some sort of serial processing, with the cuing dimension being processed first. Many models of parallel processing involving different rates for the cuing and the cued dimensions could lead to similar conclusions.

Another possibility, expressed by some subjects, is that these stimulus letters are so clear that it did not seem necessary to bother processing the cuing dimension. Such an attitude on the part of the subjects does not recognize how much faster they are on discrimination tasks than on any form of classification task. Yet if the task seems easy enough, subjects may indeed treat the cuing dimension as irrelevant, and attempt to ignore it rather than use it.

One last possible explanation lies in the basic nature of the contingent task. Even if the cuing information is fully used, the result is that the subject is randomly alternating between two different discrimination tasks, and this intermixing of tasks can lead to enough deterioration of performance that it offsets the possible gain of the use of the cuing dimension.

\section{Precuing}

To clarify some of these issues, the next experiment had the same logical structure as the present one, except that, instead of using location cuing concurrently with the actual stimulus presentation, the classification tasks were precued. The precuing was not done with a dimension, so the term "contingent" was no longer appropriate, even though precuing could be done with advance location information, for example. Rather, the precuing was simply the presentation of the task and response assignments immediately prior to the presentation of the actual stimulus. With this technique, the discrimination tasks would still be randomly alternating, but information about the specific task would be given sufficiently early for there to be no need to trade off processing time for a cuing dimension with that for the stimulus proper.

\section{EXPERIMENT 4: PRECUING}

\section{Subjects}

\section{Method}

Seven subjects were recruited from Yale students and staff, and were paid for participation. All had normal or corrected vision, and all had had previous experience in this type of experiment.

\section{Tasks}

Classification. The difficult classification task used in the last two experiments was used again, CL/OT. It was used in three forms. As a control, all four stimuli appeared randomly, with two stimuli requiring each of the two responses. In two precued classification tasks, all four stimuli still appeared randomly, but before each stimulus presentation a pair of letters was shown to indicate that on this particular trial the stimulus would be one of these two. Thus, on a trial-by-trial basis the subject was presented with a discrimination task involving just two stimuli, with two different discrimination tasks randomly alternated. Two sets of discrimination pairs were used. In one precued classification, the discrimination tasks were easy. $\mathrm{C} / \mathrm{T}, \mathrm{L} / \mathrm{O}$. In the other precued task, the discrimination tasks were hard, $\mathrm{C} / \mathrm{O}, \mathrm{L} / \mathrm{T}$. Thus, all three classification tasks were hard. but one of the precued tasks led to easy discriminations and the other to hard discriminations.

Discrimination. Four discrimination tasks were used, corresponding to the easy and hard discriminations used in the precued classifications. The easy tasks were $\mathrm{C} / \mathrm{T}$ and $\mathrm{O} / \mathrm{L}$; the hard discriminations were $\mathrm{C} / \mathrm{O}$ and $\mathrm{L} / \mathrm{T}$.

For both types of task, the stimulus was centered, with no surrounding field other than the white background of the card. 


\section{Stimulus Presentation}

Timing. Between trials, a blank card was exposed without response assignments or a fixation dot. When a subject initiated a trial, the cue card appropriate for that trial appeared and remained for $500 \mathrm{msec}$, at which time the stimulus appeared for the duration of the stimulus exposure. The field then reverted to the blank card.

Cue cards. The cue cards used were identical to the reminder cards used in Experiment 1, including the fixation. When the task was control classification, two horizontally separated stimuli appeared above and two appeared below the fixation dot to indicate the response assignment. With both precued classification and discrimination tasks, the cue cards contained just two horizontally centered stimuli, one above and one below to indicate the response assignment. In the discrimination tasks and control classification, the same cue card appeared on every trial of the block of trials for that condition. In the cued classification tasks, the two cue cards appropriate for that task randomly alternated within the blocks of trials. Response assignments (up or down) were never varied for a single block of trials.

\section{Experimental Design}

All seven tasks were used in each session. Before each task was started, 12 practice trials were given to be sure the subject understood the nature of the particular task. Then, for each task, 18 trials were given per stimulus. Thus there were 72 total trials for all three classification tasks and $\mathbf{3 6}$ trials for each discrimination task.

The seven tasks were run with a Latin square counterbalancing. Each subject was run for two sessions with the same Latin square, but data were used from the second session only.

\section{Results}

The results are shown in Table 5. As in Experiment 3, the data for the two easy discrimination tasks were averaged, as were those for the two hard discrimination tasks. Such averaging allows direct comparison with the equivalent precued tasks, and the numbers of trials for the discrimination and the classification tasks are made comparable.

\section{Classification Tasks}

An initial overall ANOVA was carried out for the five tasks shown in Table 5, and as is evident from these data, tasks were a significant source of variance $[\mathrm{F}(4,24)=$ $18.01, \mathrm{p}<.001]$. However, of greater importance are the effects within and between the two types of task.

A contrast between the control classification and the easy precued task, $\mathrm{C} / \mathrm{T}, \mathrm{L} / \mathrm{O}$, with a difference of $50 \mathrm{msec}$,

\section{Table 5}

Experiment 4: Reaction Times for Control Classification, Precued Classification Tasks, and the Equivalent Discrimination Tasks

\begin{tabular}{ccc}
\hline Task Type & $\begin{array}{c}\text { Specific } \\
\text { Task }\end{array}$ & $\begin{array}{c}\text { Mean RT } \\
\text { (msec) }\end{array}$ \\
\hline Classification & & \\
Control & CL/OT & 409 \\
Precued & C/T,L/O & 359 \\
Precued & C/O,L/T & 379 \\
Discrimination & C/T and O/L & 343 \\
& C/O and L/T & 368 \\
\hline
\end{tabular}

Note-The comma separates the two components of the classification tasks that are precued. RTs are averaged for the pairs of discrimination tasks that correspond to the equivalent precued classification tasks. was significant $[\mathrm{F}(1,24)=36.94, \mathrm{p}<.001]$. A contras between the control classification and the hard precued task, C/O,L/T, with a difference of $30 \mathrm{msec}$, was also significant $[F(1,24)=12.97, p<.01]$. Thus, the use of precued classification resulted in improved performance for both precued tasks.

However, there was also a significant difference of $20 \mathrm{msec}$ between the easy and hard precued classifications $[\mathrm{F}(1,24)=6.13, \mathrm{p}<.025]$, and since this effect is in the expected direction, a one-tailed significance test is more appropriate. Thus, the use of precuing not only improves classification performance overall, but it also provides an amount of improvement appropriate to the difficulty of the discriminations produced by the precuing.

\section{Discrimination Tasks}

The two pairs of discrimination tasks differed from each other by $25 \mathrm{msec}$ in the direction expected, namely that performance was poorer for the hard discrimination tasks $[F(1,24)=9.51, p<.01]$. This result duplicates that of Experiment 1, as well as that of Experiment 3, and was fully expected.

The reason for using these tasks was to provide controls for determining how much improvement was obtained by precuing the classification task. If precuing was completely effective, then the precued tasks should have given the same results as their equivalent discrimination tasks. In the case of the hard discriminations, the difference of $11 \mathrm{msec}$ faster for the discrimination tasks was not significant; nor was the difference of $16 \mathrm{msec}$ in favor of the easy discrimination tasks significant. However, when these two contrasts are combined into a single contrast comparing the mean of $369 \mathrm{msec}$ for the precued classification tasks and the mean of $356 \mathrm{msec}$ for the discrimination tasks, significance was obtained $[F(1,24)=$ $5.25, \mathrm{p}<.05$ ]. Thus, this average difference of $13 \mathrm{msec}$ indicates that the precued tasks were not quite as effectively performed as their equivalent discrimination tasks, but the difference is sufficiently small to state that precuing was very effective.

Further evidence that precuing produced nearly the same performance as the discrimination tasks is that the difference between the hard and easy precued tasks was $20 \mathrm{msec}$, a figure very close to the same difference of $25 \mathrm{msec}$ for the discrimination tasks. Thus, the overall picture is that precuing by 500 msec makes the classification tasks very much like their equivalent discrimination tasks.

\section{Discussion}

The results of the precuing experiment indicate that there is no inherent problem with the contingent classification that cannot be resolved if the cuing information is provided in advance. The fact that the precuing was done by directly specifying the stimuli on each trial is not a relevant consideration, since advance color or location information could have been used just as well, but it simply would have added a memory problem unnecessarily. 
In fact, Miller (1983), in a comparable experiment, did cue location with an arrow, and obtained improvement in performance compared with the control or uncued classification.

The other issue that this experiment addressed was whether the failure to obtain reasonably large performance gains with the contingent task was due to the random intermixing of what are the equivalent discrimination tasks. The same random intermixing occurred in this experiment that occurred in the two preceding ones, and there was a substantial gain in performance. However, there was an average difference of $13 \mathrm{msec}$ between the precued.tasks and the discrimination tasks, and this difference might well indicate that there is some small amount of performance decrement in the precued (or contingent) tasks due to the random intermixing of discrimination trials.

\section{GENERAL DISCUSSION}

Discussion of the findings of each experiment was presented after the results for each experiment, so no further detailed discussion is necessary. There are, however, some broader theoretical issues about contingent and precued classification that need comment.

\section{Parallel or Serial Processing}

Although the distinction between serial and parallel processing has become increasingly blurred in recent years, the difference between them is still useful as a framework for discussion of many results, including those presented here. The logical structure of the contingent classification task is, in fact, one that seems to call for serial processing with discrete stages: first the cuing dimension is processed, and the outcome of that process then determines what other processing need take place. The complete failure to obtain any effectiveness of contingent cuing with color, plus the small effectiveness with location cuing, and then only when the cued discrimination tasks were easy, makes clear that no such discretestage processing occurred. When, however, the cuing information was given in advance, the results were quite like those one would expect with simple serial processing. Thus, it would appear that such serial processing can be used only when the timing of the processing is determined by the timing of the presentation of the two kinds of information.

A more reasonable expectation for the processing of contingent classification would seem to lie in a model of some form of processing that assumes a continuous flow of information from two or more sources, models such as the continuous-flow model of Eriksen and Schultz (1979), the cascade model of McClelland (1979), or Turvey's (1973) concept of concurrent-contingent processing. What all of these have in common is their assumption that processing of the cuing and the contingent dimensions would go on concurrently, but if processing of the cuing dimension were sufficiently faster than processing of the contingent dimension, there should be an improvement in classification speed when a cuing dimension is added. Once again, the results with concurrent cuing showed effectiveness only with location cuing, and then only when the cued discrimination task was easy. This last result is actually contrary to what these models should predict, because if the contingent task is hard, processing of the contingent dimension should be slow relative to processing of the cuing dimension, and a greater gain should obtain for the contingent dimension than if its processing were easy.

\section{Attentional Strategies}

Very possibly, as Pomerantz and Sager (1975) remarked when trying to make similar distinctions, asking what form of serial or parallel processing is correct for these tasks is simply inappropriate. As Kahneman (1973) has also suggested, to understand performance in such tasks it is more important to consider the role of attention to the different dimensions, and the strategies invoked by subjects in allocating attention to these dimensions. As I have also noted (Garner, 1970), subjects are not fixed to a single strategy, serial or parallel processing, or even as the later authors noted, to strategies involving allocation of attention. Rather, subjects can alter their processing strategies to accomplish what would seem to them to be some optimization of performance.

With the color-cuing experiment, subjects apparently allocated all of their attention to the letters and no attention to the colors. As noted earlier, some subjects commented that they simply ignored color. Yet there was a potential gain for the difficult classification tasks of about $100 \mathrm{msec}$, given the base data of Experiment 1. Why did it not seem worth allocating some attention to the cuing dimension? The answer most probably lies in the subject's assumption, whether true or false, that to allocate some attention to color would produce some deterioration in letter-classification performance, since less attention would be allocated to the contingent dimension.

That such an assumption might be valid is suggested by the data with location cuing. Note that performance was improved only when the cued discriminations were easy, even though that is the opposite of what would be expected on a serial time basis. If, however, those tasks were sufficiently easy for some attentional resource to be allocated to the cuing dimension, then the subject could afford to distribute attention over the two types of dimension and achieve some gain in performance. That no equivalent result occurred with color cuing simply suggests that location requires less attention than does color, that is, is a more effective cuing dimension.

What is clear is that neither color nor location demanded attention so as to remove the option, from the subject, of not allocating attention to it. Whether or not there are any dimensions that require mandatory attention cannot be known from these data, but color and location are such strong candidates for mandatory attention that it seems unlikely that there are any cuing dimensions to which subjects must attend. Of course, with precuing, the attentional 
resources do not have to be distributed, and subjects in that case can make full use of the precuing information.

To summarize, the most reasonable explanation of these data is that subjects simply did not choose to allocate attention to the cuing dimension, except in the one case in which attentional demands for the easy discriminations with location cuing made it possible to allocate attention to location with little or no attentional cost in dealing with the easy discriminations.

\section{Levels of Difficulty}

The letters used in the present experiment were very clear and basically easy to discriminate, as were also the levels on the cuing dimensions. Suppose that the contingent dimension were made much more difficult to process. Would this force or entice the subject to allocate a greater amount of attention to the cuing dimension, even though the contingent dimension itself would demand more attention because of its greater difficulty? It seems likely that it would, since now a sufficient gain could be obtained at small relative cost in attentional allocation. The results of Miller (1983) showed that the value of precuing for response was greater when the task was difficult than when it was easy, so it seems reasonable that an equivalent result would be obtained if the contingent task was increased in difficulty, while still using very discriminable cuing dimensions.

If such a result were obtained, it would not differentiate between serial and continuous flow (parallel) models, because these conceptions of processing would both expect earlier information from the cuing dimension, thus making it more available to cue the contingent dimension. However, from the results of the present experiments alone, such a failure to differentiate between serial and any form of parallel processing would not be important, since the best explanation of these results lies in understanding different strategies of attentional allocation.

Response cuing or stimulus cuing? The primary intent of the Miller (1983) study was to investigate the use of precuing in response preparation (see also Miller, 1982, 1985), yet stimulus factors such as stimulus discriminability influenced the effectiveness of the precuing. Furthermore, Reeve and Proctor $(1984,1985)$ argued that the results obtained by Miller were not simply due to motoric factors, but that decision processes occurring prior to response preparation were involved. While this argument for decision processes is more specific to an ultimate response preparation, it is not unlike the argument here that attentional strategies are involved in the use of precued information. In both cases, the argument is that the organism has strategic options about the processing of stimuli to be identified or classified. However, in the present experiments, cuing or precuing did not alter response assignments, so it is clear that the strategies do not involve direct response preparation. The use of the contingent classification paradigm thus provides one direct experimental procedure for clarifying some of these issues.

\section{REFERENCES}

Biederman, I. (1972). Human performance in contingent informationprocessing tasks. Journal of Experimental Psychology, 93, 219-238.

Duncan, J. (1981). Directing attention in the visual field. Perception \& Psychophysics, 30, 90-93.

Eriksen, C. W., \& Schultz, D. W. (1979). Information processing in visual search: A continuous flow conception and experimental results. Perception \& Psychophysics, 25, 249-263.

GARNER, W. R. (1970). The stimulus in information processing. American Psychologist, 25, 350-358.

GaRnER, W. R. (1974). Attention: The processing of multiple sources of information. In E. C. Carterette \& M. P. Friedman (Eds.), Handbook of perception (Vol. 2, pp. 23-59). New York: Academic Press.

GARNER, W. R. (1980). Functional aspects of information processing. In R. S. Nickerson (Ed.), Attention and performance VIII (pp. 1-26). Hillsdale, NJ: Erlbaum.

GARNER, W. R. (1983). Asymmetric interactions of stimulus dimensions in perceptual information processing. In T. J. Tighe \& B. E. Shepp (Eds.), Perception, cognition, and development: Interactional analyses (pp. 1-38). Hillsdale, NJ: Erlbaum.

HoDGE, M. H. (1959). The influence of irrelevant information upon complex visual discrimination. Journal of Experimental Psychology, $57,1-5$.

Kahneman, D. (1973). Attention and effort. Englewood Cliffs, NJ: Prentice-Hall.

LaBerge, D., Van Gelder, P., \& Yellott, J. (1970). A cueing technique in choice reaction time. Perception \& Psychophysics, 7, 57-62.

MCClelland, J. L. (1979). On the time relations of mental processes: An examination of systems of processes in cascade. Psychological Review, 86, 287-330.

MiLlER, J. (1982). Discrete versus continuous stage models of human information processing: In search of partial output. Journal of Experimental Psychology: Human Perception and Performance, 8, 273-296.

Miller, J. (1983). Can response preparation begin before stimulus recognition finishes? Joumal of Experimental Psychology: Human Perception and Performance, 9, 161-182.

MiLLER, J. (1985). A hand advantage in preparation of simple keypress responses: Reply to Reeve and Proctor (1984). Journal of Experimental Psychology: Human Perception and Performance, 11, 221-233.

Montague, W. E. (1965). Effect of irrelevant information on a complex auditory-discrimination task. Journal of Experimental Psychology. 69. 230-236

Pomerantz, J. R., \& SaGer, L. C. (1975). Asymmetric integrality with dimensions of visual pattern. Perception \& Psychophysics, 18, 460-466.

PoSNER. M. I. (1978). Chronometric explorations of mind. Hillsdale, NJ: Erlbaum.

ReEve, T. G., \& Proctor, R. W. (1984). On the advance preparation of discrete finger responses. Journal of Experimental Psychology: $\mathrm{Hu}$ man Perception and Performance, 10, 541-553.

Reeve, T. G., \& Proctor, R. W. (1985). Nonmotoric translation processes in the preparation of discrete finger responses: A rebuttal of Miller's (1985) analysis. Journal of Experimental Psychology: Human Perception and Performance, 11, 234-241.

RUTH, D. S. (1976). The effects of pattern goodness on automatic and strategy-dependent processes. Unpublished doctoral dissertation, Yale University.

SHAFFER, L. H. (1965). Choice reaction time with variable S-R mapping. Journal of Experimental Psychology, 70, 284-288.

TSAL, Y. (1983). On interpreting the effects of location preknowledge: A critique of Duncan. Perception \& Psychophysics, 34, 297-298.

TuRVEY, M. T. (1973). On peripheral and central processes in vision: Inferences from an information-processing analysis of masking with patterned stimuli. Psychological Review, 80, 1-52.

VON WRIGHT, J. M. (1968). Selection in visual immediate memory. Quarterly Journal of Experimental Psychology, 20, 62-68.

(Manuscript received May 30, 1985; revision accepted for publication August 29, 1985.) 